\title{
Stellar Populations of Halo Substructure Along The Major Axis of M31
}

\author{
J. C. Richardson ${ }^{1}$, A. M. N. Ferguson ${ }^{1}$, R. A. Johnson ${ }^{2}$ and D. C. Faria ${ }^{3}$ \\ ${ }^{1}$ Institute for Astronomy, University of Edinburgh, Blackford Hill, Edinburgh, UK, EH9 3HJ \\ email: jcr@roe.ac.uk \\ ${ }^{2}$ Department of Astrophysics, University of Oxford, Keble Road, Oxford, UK, OX1 3RH \\ ${ }^{3}$ Institute of Astronomy, University of Cambridge, Madingley Road, Cambridge CB3 0HA
}

\begin{abstract}
We present HST/ACS observations of five regions in the outskirts of M31. Four are centered on prominent stellar overdensities roughly aligned with the major axis. Well-populated colour magnitude diagrams (CMDs) show that these fields differ in morphology from the fifth (Minor Axis) field. Comparisons with isochrones present tantalising evidence that they contain a small contingent of very young $(\leqslant 1 \mathrm{Gyr})$ stars, at odds with what may be expected of typical halo populations, suggesting that this low-latitude substructure could feasibly have formed in the disk.
\end{abstract}

Keywords. galaxies: individual (M31), galaxies: halos, galaxies: stellar content, galaxies: evolution

\section{Introduction}

The stellar halos of massive galaxies are predicted to contain evidence of past mergers and accretion events in the form of tidal debris and substructure (Bullock \& Johnston (2005)). In addition, satellite accretions will heat, thicken and even disrupt the stellar disk providing another mechanism capable of producing low-latitude substructure (Gauthier et al. (2006)). M31 is known to have an abundance of halo stellar substructure (Ferguson, et al. (2002)). Deep HST/ACS photometry targeted on 6 locations of exceptional interest exhibit morphological variations between most of the CMDs (Ferguson et al. (2005)) suggesting the substructure is not the product of a single accretion event. Two new features have been observed and all the data reduced consistently with stand-alone DAOPHOT II (Stetson (1987)). This contribution focuses on a subsample of these fields located along the major axis.

\section{Results}

Figure 1 (top panel) shows Hess diagrams of the major axis fields (the G1 Clump, N Spur, NE Clump and Claw) and the control field (the Minor Axis). The reader is referred to Ferguson et al. (2005) for their positions with respect to M31. The NE Clump and Claw are more recent observations situated $\sim 40 \mathrm{kpc}$ (projected) to the northeast and $\sim 24 \mathrm{Kpc}$ to the southwest of M31 respectively. The former is the furthest substructure to be imaged. All CMDs have a broad RGB and prominent red clump (RC) suggesting a large metallicity spread and bulk intermediate age population. However, while the Minor Axis field has a faint blue HB indicating the presence of an old, metal poor population, the major axis fields display blue plumes (BP) associated with young main-sequence stars. The extent of the BP varies between fields reaching $\sim 23.2 \mathrm{mag}$ in the G1 Clump. Around $10 \%$ of the mass of this population is believed to have formed in the last 2 Gyr (Faria et al. (2006)). Isochrones with $[M / H]=-0.4$ dex and ages $\leqslant 1$ Gyr agree well with the shape of the BP. It is particularly remarkable that such a young population is seen in the NE Clump which sits out at $40 \mathrm{kpc}$, well beyond the main HI disk.

The major axis luminosity functions (LF) are strikingly similar in morphology, peak magnitude and spread (figure 1, bottom panel), visibly differing from the Minor Axis LF. RC magnitude and colour vary with age and metallicity, with an especially strong dependence for 

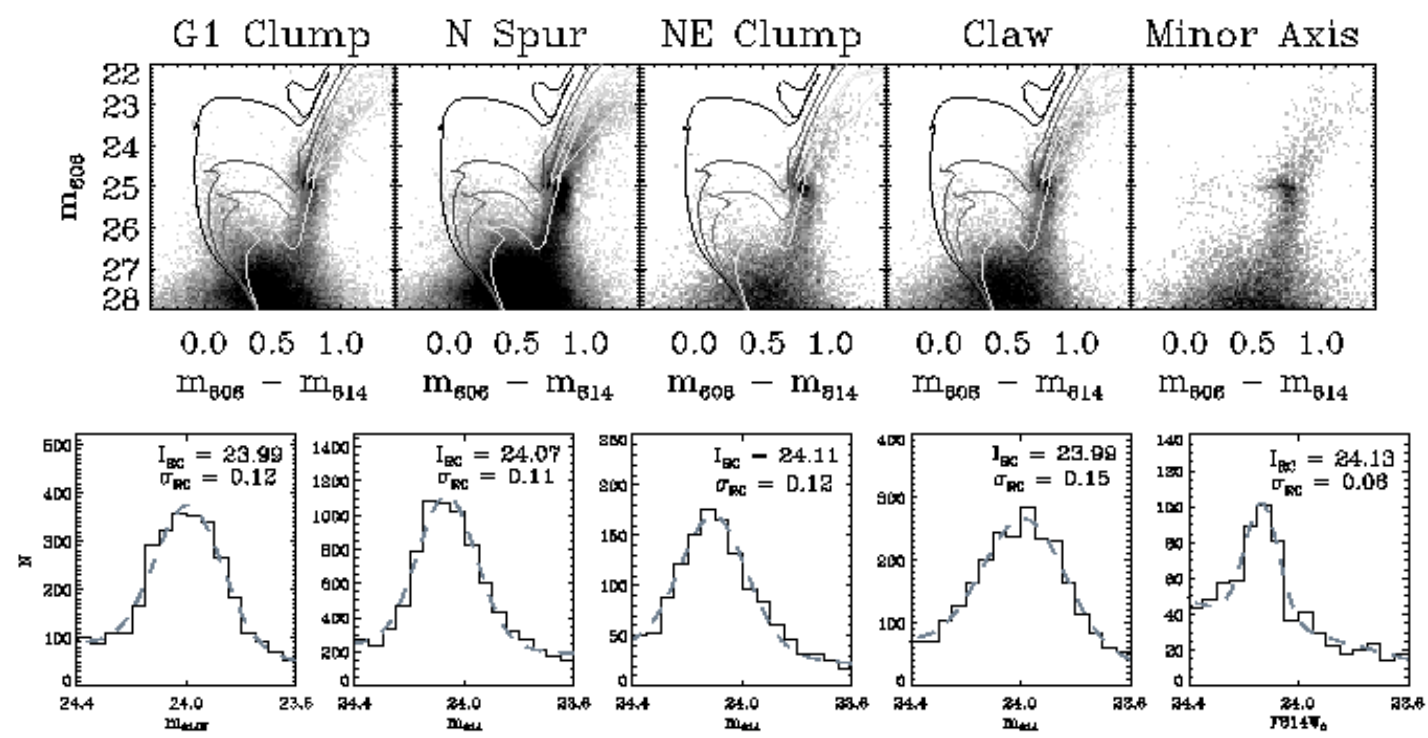

Figure 1. Top Panel: Hess diagrams of the major axis halo fields compared to isochrones of $[M / H]=-0.4$ dex and ages 250,630, 1000 and 1800 Myr left to right (Girardi et al. (2000)). The Minor Axis field has a faint blue horizontal branch rather than a blue plume. Bottom Panel: Gaussian fits to the $m_{814}$ RC luminosity function (order as above). Magnitudes are in the VEGAmag system.

stars younger than $\leqslant 3$ Gyr (Girardi \& Salaris (2001)). It is likely that the greater spread in the RC LF of the major axis fields is a manifestation of their having a broader age range.

\section{Discussion}

Although the major axis fields vary in the extent and relative density of their BP from field to field, all possess young, metal rich populations in sharp contrast to the Minor Axis field, our other halo substructure fields (Ferguson et al. (2005)) and to the extremely deep spheroid and stream fields of Brown et al. (2006). They are also unlike any present day dwarf spheroidal companion of M31 (Da Costa et al. (2002)). The major axis fields appear consistent with having formed in the disk. If correct, this would provide a natural explanation for the fact that much of the low-latitude substructure around M31 rotates with the disk (Ibata et al. (2005)).

\section{Acknowledgements}

We wish to acknowledge Mike Irwin, Rodrigo Ibata, Kathryn Johnston, Geraint Lewis and Nial Tanvir for their continued collaboration on this work.

\section{References}

Brown, T. et al. 2006, ApJ, 652, 323

Bullock, J. \& Johnston, K. 2005, ApJ, 635, 931

Da Costa, G., Armandroff, T., \& Caldwell, N. 2002, AJ, 124, 332

Faria, D. et al. 2006, astro-ph/0611852

Ferguson, A., Irwin, M., Ibata, R., Lewis, G., \& Tanvir, N. 2002, AJ, 124, 1452

Ferguson, A. et al. 2005, ApJ, 622, L109

Gauthier, J.-R., Dubinski, J., \& Widrow, L. 2006, ApJ, 6531180

Girardi, L., Bressan, A., Bertelli, G., \& Chiosi, C. 2000, A\&AS, 141, 371

Girardi, L. \& Salaris, M. 2001, MNRAS, 323, 109

Ibata. R. et al. 2005, ApJ, 634, 287

Stetson, P. 1987, PASP, 99, 191 